Aus der Universitäts-Frauenklinik zu Heidelberg. - Director: Prof. Dr. C. Menge.

\title{
Die geeignetsten Methoden zur Untersuchung des Lochialsecretes auf aerobe und anaerobe Strepto- kokken, ihre Resultate und klinische Bewerthung.
}

\author{
Von \\ Dr. F. Goldschmidt, \\ Assistent der Klinik.
}

Seitdem Pasteur den Streptococcus pyogenes als den hauptsächlichsten Erreger des Wochenbettfiebers nachgewiesen, ist es das unausgesetzte Bestreben der Geburtshelfer gewesen, die Diagnose und Prognose des Puerperalfiebers auf eine gesicherte wissenschaftliche Grundlage zu stellen.' Die überaus zahlreichen Arbeiten der letzten Jahrzehnte liefen daher vor Allem darauf hinaus, über die Bakterienflora des weiblichen Genitaltractus und über das Vorkommen der verschiedenen krankmachenden Erreger im Genitaltractus in Schwangerschaft und. Wochenbett Klarheit zu schaffen. Man bemühte sich besonders festzustellen, ob man auf Grund zuverlässiger Differencirung erkennen kann, ob in der Bakterienflora einer Schwangeren, Kreissenden oder Wöchnerin der gefundene Streptococcus nur als harmloser Saprophyt oder als Keim des so häufig letal endigenden Puerperalfiebers lebt.

Seit der grundlegenden Arbeit von $\mathrm{Menge}$ und Krönig auf diesem Gebiete sind durch die Einführung von Neuerungen und Verbesserungen in die Reihe der bakteriologischen Untersuchungsmethoden auch deren Resultate in einigen Punkten modificirt worden. Hierzu trug besonders zum Nachweis der Streptokokken die Einführung des flüssigen Nährbodens von Walthard und der Blatagarplatte durch Schottmüller bei, mit denen einige Untersucher selbst in der Scheide Hochschwangerer in 70, ja 100 pCt. der Fälle Streptokokken und unter diesen in einem grossen Procentsatz angeblich den Streptococcus haemolyticus gefunden haben wollen. 
286 Goldschmidt, Methoden zur Untersuchung des Lochialsecretes u.s.w.

Auch suchte man die Fortschritte, die man so im Wesentlichen durch Verfeinerung der Untersuchungsmethoden erzielt hatte, für die Frage der Entscheidung nach der Arteinheit oder Artvielheit der Streptokokken, nach ihrer Pathogenität oder Apathogenität zu verwenden. So glaubte man mit Hilfe der Hämolyse und Blutagarplatten eine Differencirung vornehmen zu können in hämolytische pathogene und nichthämolytische apathogene Streptokokken, wie dies zuerst von Schottmüller und später mit besonderem Eifer von der Hallenser Schule geschehen ist.

Bei allen diesen Untersuchungen stellte es sich immer mehr heraus, dass zur Erzielung correcter Resultate nicht nur die Verwendung der Nährböden, sondern auch eine Reihe von Technicismen zu beachten sind, und dass die grossen Differenzen in den Resultaten vieler Arbeiten in deren Ausserachtlassen zu suchen sind.

So gelang es z. B. einem so geübten und erfahrenen Untersucher wie Fromme zunächst niemals, bei nicht fiebernden normalen Wöchnerinnen hämolytische Streptokokken nachzuwejsen. Er fand dieselben nur bei fiebernden Wöchnerinnen und zog aus dieser Thatsache die allerweitest gehenden Folgerungen. Schon bald darauf musste er zugeben, dass, wie bereits vorher Sigwart, so später sein Mitarbeiter Heynemann mit gegen früher abgeänderten Methoden der Aussaat des zu untersuchenden Materiales öfters in der Scheide, im Cervicalsecret und auch im Uterus bei normalen Wöchnerinnen Streptokokken mit typischer Hämolyse im Ausstrich und auch in der Mischoultur fand:

Und noch aus den Verhandlungen des letzten Gynäkologencongresses zu Strassburg geht hervor, dass in der Beurtheilung der zweckmässigsten Art und Weise der Verarbeitung des Lochialsecretes mehrfach unter den verschiedenen Forschern Differenzen bestehen, und dass eine Methode, die dem einen als aussehlaggebend und beweisend gilt, von anderer Seite bekämpft und verworfen wird. Noch grössere Differenzen aber als in der Feststellung und Sicherung der bakteriologischen Diagnose des Puerperalfiebers treten zu Tage in der prognostischen Werthung und Deutung der durch die bakteriologische Untersuchung erzielten Resultate.

Ich habe es mir daher bei meinen Untersuchungen des Lochialsecretes von fiebernden und nichtfiebernden Wöchnerinnen, dic ich im letzten Jahre auf Anregung von Herrn Prof. Menge ausgeführt habe, angelegen sein lassen, die verschiedensten angegebenen Unter- 
suchungs- und Culturmethoden nebeneinander in grösseren Serien vorzunehmen, um festzustellen, welche Methoden die besten und einwandfreisten Ergebnisse liefern, d. h. sich am besten mit den klinischen Erscheinungen decken, und wo die Schwächen und Fehlerquellen der einzelnen Methoden liegen.

Dabei habe ich mein Hauptangenmerk auf den Streptococcus in seinen verschiedenen Formen, in seinen tinctoriellen, morphologischen und culturellen Eigensehaften gerichtet, da er erwiesenermaassen der weitaus häufigste Erreger des Puerperalfiebers ist.

Aus der Reihe der Fragen, die ich durch meine Untersuchung beantworten wollte, seien nur kurz einige wenige angedeutet.

So lag mir daran, festzustellen, ob die bisher geübte Secretentnahme aus dem Uterus ersetzt werden kann durch die von Heynemann empfohlene Secretentnahme aus der Scheide. Ob die Behauptung von Sigwart zu Recht besteht, dass man schon in den meisten Fällen aus dem einfachen Ausstrichpräparat die Diagnose zu stellen vermöge, und dass es zu bedauern sei, dass dieses werthvolle Hilfsmittel, das doch für den Praktiker das einzige leicht erreichbare, in der letzten Zeit leider zu sehr in den Hintergrund getreten sei. Ferner wollte ich über die absolute und relative Verwerthbarkeit der flüssigen und festen Nährböden Aufschluss gewinnen. Haben doch Krönig und Pankow sich erneut für die Verwendung der schwach alkalischen Agargussplatte ausgesprochen, die nach ihrer Meinung sogar für die Stellung der Diagnose gewisse Vortheile bieten soll.

Andere Untersucher, wie Zangemeister und Sachs, sind in der letzten Zeit besonders lebhaft für den Oberflächenausstrich auf der Blutagarplatte eingetreten, da ihnen dieses Verfahren werthvoller erschien, als das der Blutagargussplatte. Sachs behauptet sogar, dass eine Infection mit hämolytischen Streptokokken nur dann vorliegt, wenn in Krankheitsfällen diese Keime beim Ausstrich einer Oese Lochialsecret oberfläehlich auf der Blutplatte fast in Reincultur aufgehen oder doch in nicht zweifelhafter Mehrzahl aller Colonien.

Unter Berücksichtigung dieser verschiedenen Punkte habe ich die Anordnung meiner Versuche aufgestellt.

Zunächst möchte ich einige recht wesentliche Angaben über die Art der Entnahme und Verarbeitung des Lochialsecretes machen, 
da gerade diesem Punkt anerkannter Maassen eine sehr grosse Bedeutung zukommt und sich in den letzten Jahren darin eine sehr wesentliche Veränderung vollzogen hat.

Die bisher allgemein übliche Weise der Secretentnahme gestaltete sich nämlich folgendermaassen:

Die Wöchnerin wurde auf dem Querbett oder auf dem Untersuchungsstuhl gelagert. Nach Einstellen der Portio mit sterilen Speculis wurde dieselbe zunächst mit sterilen Wattetupfern von anhaftendem Secret gereinigt und alsdann ein Walthard'scher Pinsel oder ein Döderlein'sches Secretröhrchen bezw. ein Röhrchen ähnlicher Construction, das an seinem einen Ende mit dem Finger versehlossen gehalten wurde, in den Uterus eingeführt. Mittelst eines Saughütchens oder einer Luftpumpe wurde das Secret dann angesaugt, wenn es nicht von selbst, wie häufig, in das Röhrchen hineinfloss. Das Röhrchen wurde dann an beiden Enden zugesiegelt und verarbeitet, indem von der erhaltenen Secretsäule nur der mittelste Theil verwendet wurde.

Dieses sehr umständliche und zeitraubende Verfahren hat den ausserordentlich grossen Nachtheil, dass es bei vielen Patienten, namentlich bei unruhigen Wöchnerinnen, selbst in der Klinik schwer durchführbar ist, dass es vor Allem für die Wöchnerinnen nicht. gleichgültig ist und mitunter selbst Lebensgefahr mit sich bringt, ganz abgesehen davon, dass es in der ausserklinischen Praxis so. gut wie garnicht anwendbar ist. Auch wird man bei pyämischen und thrombotischen Affectionen diese Procedur nur ungern vornehmen wollen. Andererseits läuft man Gefahr, bei nicht diagnosticirten thrombophlebitischen Processen die übelsten Zufälle zu erleben. So berichtet Heynemann, dass bei dieser Art der Secretentnahme einmal in der Hallenser Klinik trotz aller angewandten Vorsichtsmaassregeln eine Hirnembolie eingetreten sei. Auch kann diese Art der Secretentnahme bei Ungeübten leicht zu Verletzungen und ernsten Schädigungen der Kranken Veranlassung geben.

Es ist nämlich nicht von der Hand zu weisen, dass auch durch Verschleppung Keime (die vorher nur in der Scheide waren) bierbei erst in den Uterus hineingebracht werden können. Ferner ist es sicherlich nicht gleichgültig, ob durch die zahlreichen Manipulationen kleine verheilte Wunden in der Scheide oder im Uterus wieder aufreissen, neue gesetzt und eventuell virulente Keime dadurch in die tieferen Gewebsschichten; in die Blut- und Lymphbahnen ge- 
drückt werden, und so Keimen, die bisher oberflächlich lebten, eine grössere Agressivität gegenüber dem Organismus verliehen wird.

Man kann deshalb auch niemals mit Sicherheit entscheiden, ob ein im Anseblass an die Secretentnahme einsetzendes Fieber oder eine Versehlimmerung des Zustandes nicht unterblieben wäre, wenn man diesen Eingriff nicht vorgenommen hätte, und ob man so nicht ans dem "Fintagsfieber" eine längerdauernde Infection gemacht hat.

Aus allen diesen schwerwiegenden Gründen, die gegen eine Secretentnahme aus dem Uterus sprechen, war es lebhaft zu begrüssen, als Heynemann, fussend auf einer grossen Anzahl Untersuchungen von Schottmüller und Mau über den Keimgehalt der Scheide und des Cervicalcanales bei Schwangeren an hämolytischen Streptokokken, darauf hinwies, dass es zum Nachweis des Streptococcus haemolyticus völlig genüge, das Secret bereits aus dem vordersten Theil der Scheide mit einer Platinöse oder einem Wattebausch zu entnehmen. Es ergab sich somit für ihn das höchst einfache folgende Verfahren:

Bei der ruhig im Bett liegenden Wöchnerin werden die Labien auseinandergespreizt, mit einer ausgeglühten Platinöse oder sterilem Wattebausch wird etwas Secret aus dem vorderen Theil der Scheide entnommen und fein auf der Schottmüller'schen Blutagarplatte $2: 5$ ausgestrichen. Die Platten werden in den Brutschrank gebracht und nach 7-10-12 Stunden wird die Diagnose abgelesen. Durch zahlreiche vergleichende Untersuchungen des Uterus- und Vaginalsecretes und dureh Vergleich der klinischen mit den bakteriologischen Befunden konnte Heynemann die Vollwerthigkeit dieser Methode, soweit sie den hämolytischen Streptococeus betrifft, mit der directen Entnahme aus dem Uterus sicher stellen.

Sachs; der sich zu seinen Untersuchungen ebenfalls dieser einfachen Methode bediente, macht nur die Angabe, dass in der Verarbeitung des Scheidensecretes anstatt des Uterussecretes nach seinen Erfahrungen kein Nachtheil liegt. Es ist ihm nie vorgekommen, dass pathogene Keime ihm dadurch entgangen sind, die sich dann eventuell später in der Leiche nachweisen liessen.: Bei weit über 200 Fällen hat die Methode bei ihm nicht versagt.

Sigwart untersuchte zur Controle die Uterus- und Vaginallochien von 12 fiebernden Wöchnerinnen. In 4 Fällen fand er weder in der Scheide, noch im Uterus Streptokokken. Dreimal 
waren allerdings die Uteruslochien steril, während aus der Scheide massenhaft hämolytische Streptokokken aufgingen. In fünf Fällen dagegen fanden sich sowohl in der Seheide als auch im Uterus typische hämolytische Streptokokken. Immerhin ist diese Differenz, die Sigwart zwischen Scheiden- und Uteruslochien fand, bemerkenswerth.

Zangemeister war auf Grund des bakteriologischen Befundes des Scheidensecretes zu der Ueberzeugung gekommen, dass zwischen diesem und der Genitalinfection klare Beziehungen bestehen, welche ätiologische Rückschüsse gestatten. In 25 aufeinander folgenden Fieberfällen, unter denen sich auch schwere Infectionen befanden, überzeugte er sich daher, wie sich der bakteriologische Befund der Scheide zu dem des Uterus verhielt, indem er beide Secrete untersuchte.

Mit einer Ausnahme konnten jedes Mal die Erreger auch in der Scheide und zwar in gleicher Qualität und Menge nachgewiesen werden. Nur in einem Fall, wo es sich offenbar um eine länger dauernde Behinderung des Lochialabflusses handelte, fand er im Uterus zahlreiche hämolytische Streptokokken, in der Scheide aber nur ahämolytische Streptokokken. Zweimal fanden sich in der Scheide Keime, obwohl der Uterusinhalt steril war. Es handelte sich dabei einmal um spärliche ahämolytische Staphylokokken.

lch habe mit der weiter unten zu schildernden Versuchsanordnung nun ebenfalls bei 40 theils fiebernden, theils nicht fiebernden Wöchnerinnen, darunter mehrere schwere und tödtlich verlaufende Fälle, Uterus- und Vaginallochien nebeneinander auf ihren Keimgehalt untersucht. Ich war dabei überrascht, zwischen Scheiden- und Uteruslochien eine weitgehende Uebereinstimmung zu finden.

Bei 25 fiebernden Wöchnerinnen der septischen Station fand ich den Streptococcus haemolyticus allein oder mit anderen Bakterien zusammen in 16 Fällen, sowohl in der Scheide als auch im Uterus, den Streptococcus viridans allein oder mit anderen Bakterien dreimal, Staphylokokken und Bacterium coli viermal, nicht weiter differencirbare Stäbchen und Kokken in zwei Fällen.

Auch bei 15 nicht fiebernden Wöchnerinnen konnte ich mich überzeugen, dass zwischen Uterus- und Vaginalsecret zum Nachweis der Streptokokken eine practisch bedeutsame Differenz nicht besteht, wenn auch in mehreren Fällen der Keimgehalt der Vaginallochien ein grösserer zu sein schien als der des Uterus. 
Die Ergebnisse dieser Untersuchungen möchte ich kurz dahin zusammenfassen, dass zwischen der Bakterienflora der Uterus- und Vaginallochien im Puerperium ein wesentlicher Unterschied gewöhnlich nicht besteht. Was die Streptokokken anbelangt, so kann man sagen, dass in allen Fällen, wo die Streptokokken im Uterus sich finden, sie auch in der Vagina nachweisbar sind, mit Ausnahme der seltenen Fälle, wo der Lochialabfluss vorübergehend gehindert wird. Umgekehrt finden sich die Streptokokken in fast allen Fällen, wo sie in der Vagina nachweisbar sind, auch im Uterus. In der Regel besteht diese Uebereinstimmung gerade bei wirklich inficirten Fällen. Nur scheinen sie im Uterus öfter bei nichtfiebernden und leicht fiebernden Wöchnerinnen in geringerer Quantität vorzukommen als in der Scheide, gelegentlich auch gar nicht. Ob dieses aber gerade bei fiebernden Wöchnerinnen in einem so hohen Procentsatz der Fall ist, wie es bei den wenigen Untersuchungen Sigwart's sich ergab, muss ich wenigstens nach meinen Untersuchungsergebnissen bezweifeln.

Practisch war für mich die Verwerthbarkeit dieser Methode trotz der nicht abzuleugnenden geringen Fehlerquellen, die ihr bisweilen anhaften, dargethan, und ich habe dann bei meinen weiteren Untersuchungen nur noch die Verarbeitung des vaginalen Secretes vorgenommen. Dieselbe geschah in ähnlicher Weise, wie sie auch von Krönig und Pankow gehandhabt wird.

leh entnahm das Secret nicht wie Fromme und Heynemann aus dem vordersten Theil der Scheide, sondern aus dem hintersten Abschnitt. Ich versprach mir so eine grössere Gewährleistung dafür, auch ein wirklich dem Uterussecret gleichwerthiges Vaginalsecret zu erhalten, ausgehend von der Ueberlegung, dass bei der weiten Verbindung des Uterus mit der Sẹheide, der Durchgängigkeit des Cervicaleanals im Wochenbett und der Gleichartigkeit der Lebensbedingungen der Bakterien im alkalischen Wochenfluss hier am sichersten die Uebereinstimmung beider Secrete zu erwarten sei. Fällt doch im Wochenbett auch die gewöhnlich bestehende bakterienfeindliche Wirkung des Cervicalsecretes fort, das sonst den Halt bildet für das Aufsteigen von Keimen.

Da es für meine Versuche darauf ankam, reichlich Secret zu erhalten, so wählte ich ein Glasröhrchen, dass in seiner Gestalt vollkommen dem Döderlein'schen Röhrchen gleicht, aber ein Lumen ron 6-8 $\mathrm{mm}$ hat. Um jegliche Verunreinigung durch Saprophyten und Keime der Vulva zu verhindern, ist das einzu- 
292 Goldschmidt, Methoden zur Untersuchung des Lachialsecretes u.s.w.

führende Ende an seinem Lumen mit einem Wattepfropf verschlossen, an dem sich ein Faden befindet, sodass man durch Zug an dem Faden den Wattepfropf ohne Weiteres herausziehen kann und das Lumen so für die Aufnahme des Secretes frei wird. Dieses Röhrchen hat sich mir auch in der poliklinischen Praxis gut bewährt und ist besonders dem Practiker zu empfehlen..

Die Secretentnahme geschieht also folgendermassen: Unter Spreizung der kleinen Labien wird das Röhrchen bis hinter den Introitus eingeführt und alsdann der vorheri etwas gelockerte Wattepfropf abgezogen. Man führt nun schnell das Röhrchen bis in den hinteren Theil der Scheide vor. Durch ganz vorsichtig hebelnde Bewegungen, die ohne Druck ausgefïhrt werden, gelingt es fast in allen Fällen, eine genügende Menge Secret zu gewinnen. Sonst kann man durch Ansaugen mit einer Luftpumpe oder einem Saughütchen sicher zum Ziele kommen. Auch die Entnahme aus dem Lterus geschah mit demselben Röhrchen.

Ueber die von mir benutzten Nährböden kann ich mich kurz fassen. Sie entsprechen der meist üblichen Zusammensetzung. Verwendet wurden:

1. 2 proc. schwach alkalische Peptonbouillon,

2. 2 proc. Traubenzuckerbouillon,

3. schwach alkalischer Agar ohne und mit Glycerinzusatz,

4. Traubenzuckeragar zu hochgeschichteten anaeroben Culturen,

5. Schottmüller'sche Blutagarplatte (2:5).

Die Bestimmung der Alkaleszenz geschah in der üblichen Weise durch Prüfung mit Lakmuspapier oder Phenolphtalein.

Die Versuchsanordnung war folgende: Es wurden mit Secret beschickt:

1. 3 Agarröhrchen mit 1, 2, 3 Normalösesecret und zu Platten gegossen,

2. 3 Blutagarplatten mit 1, 2, 3 Normalösesecret,

3. 2 hochgeschichtete Traubenzuckeragarröhrchen mit je einer Oese Secret,

4. 1 Röhrchen schwach alkalischer Bouillon mit einer Oese Secret,

5. 1 Röhrchen Traubenzuckerbouillon mit einer Oese Secret,

6. Oberflächenausstrich bei einer Schottmüller'schen Blutagarplatte, die meist kurz zuvor frisch gegossen worden war,

7. 3 Objectträgerausstriche. 
Die Controle der Culturen erfolgte nach 12, 24 und dreimal 24 Stunden. Die einzelnen Resultate wurden miteinander verglichen und protokollirt.

Von den in Bouillon gewachsenen Streptokokken wurde stets auf Agar verimpft. Die angelegten anaeroben Culturen wurden in der von Krönig angegebenen Weise verarbeitet. Das Ende des - Agarröhrchens wird an seinem geschlossenen Ende erhitzt, wodurch die Agarsänle ausgestossen wird, die man in einer sterilen Petrischale auffängt. Die Agarsäule wird alsdann in Scheiben zerlegt und aus dieser werden die einzelnen Colonien isolirt.

Zum Giessen der Sehottmüller'schen Blutplatten wurde stets steriles in einem Glaskölbchen aufgefangenes und durch Schütteln defibrinirtes Nabelschnurblut benutzt, das mir vom Geburtssaal her stets in reichlicher Weise zur Verfügung stand.

Das Anlegen der Blutagargussplatten gestattet folgende Möglichkeiten:

In den auf. $42^{\circ} \mathrm{C}$ im Reagenzrohr abgekühlten Agar bringt man die entsprechende Menge Secret, vertheilt sie fein mit der Platinöse, bringt dann Blut hinzu und giesst die Mischung in die sterile Petrischale aus. Bequemer sind die beiden folgenden, von mir mit gleich gutem Erfolg geübten Methoden: Man bringt zuerst in die Petrischale das Blut und giesst dann dazu den vorher im Reagenzrohr mit Secret beschickten Agar, sorgt für eine gleichmässige Vertheilung durch Schütteln und lässt den Agar erstarren. Oder man vertheilt das Secret mit der Platinöse in dem in die Petrischale ausgegossenen Blut und giesst dann den Agar hinzu.

Von den angefertigten Objectträger-Ausstrichen wurde je einer mit schwach alkalischer Carbolfuchsinlösung oder schwacher Methylenblaulösung und einer nach Gram gefärbt. Nach der Färbung, die sofort nach der Verarbeitung der Lochien vorgenommen wurde, habe ich mich dann stets bemüht, Anhaltspunkte für die Beurtheilung des Secrets aus dem Objectträger-Ausstrich za gewinnen und vor Allem zu sehen, wie oft und inwieweit dadurch eine Uebereinstimmung mit den culturellen und klinischen Ergebnissen zu erzielen ist.

Sigwart ist ja der Ansicht, dass man bei einer Streptokokkeninfection schon aus dem mikroskopischen Bild die Diagnose zu stellen und die bakteriologisch culturell gewonnene zu fördern vermöge. Man findet da nach seiner Meinung einen gleichmässigen 
Leukocytenteppich, dazwischen mehr oder weniger zahlreiche Diplokokken und Ketten von 4-8 Gliedern, selten mehr. Kaum das eine oder das andere Stäbchen sei zu sehen. Meist zeige sich eine rege Phagocytose, so dass das Präparat oberflächliche Aehnlichkeit mit einem frischen Gonokokkenpräparat bekomme.

Bei einem Präparat aus dem streptokokkenhaltigen Lochialsecret einer nicht fiebernden Wöchnerin soll man dagegen mehr Züge zähen homogen gefärbten Schleimes finden, zwischen denen Inseln von Leukocyten liegen. Das ganze. Präparat sei zumeist übersät von Kokken und Stäbchen aller Art in buntem Durcheinander. In einem solchen Secret könne man zerstreut zwischen den übrigen Bakterien Streptokokkenketten in grösserer Menge finden und von einer Länge, wie man sie sonst nur in künstlichem flüssigen Nährboden finde, dessen Stelle hier eben das Lochialsecret vertrete.

Wie verhält es sich nun mit dieser Behauptung? Kann man sich wirklich aus dem Ausstrichpräparat einen Rückschluss auf eine bestehende Infection gestatten?

In der von Sigwart geäusserten Form ist dieses weder für das Uterus- noch für das Scheidensecret möglich, denn dazu liegen gerade hier die Verhältnisse viel zu complicirt.

So ist es natürlich gar nicht gleichgültig, an welchem Tage man das Secret entnimmt, ob im Früh- oder Spätwochenbett, wo der Keimgehalt des Lochialsecretes ja ein ganz verschiedener ist.

Je nach dem Zeitpunkt nun, an dem die Infection einsetzt, wird man auch im Ausstrich ganz verschiedene Bilder finden. Das Bakteriengemisch wird zunächst das häufigere sein, wenn man zu Beginn der Fiebersteigerung das Secret untersucht, also zu einer Zeit, wo auch klinisch die Diagnose noch zweifelhaft ist und erst gestellt werden muss.

Die von Sigwart für die Fälle von wirklicher puerperaler Infection beschriebenen, allerdings typischen Bilder findet man selbst in Fällen sehwerster Infection nur bei einem Bruchtheil, so dass der Praktiker, wenn er sich auf dieses Kriterium allein verliesse, sehr hänfig schlimme Fehldiagnosen stellen würde.

Auch bei schwerster Streptokokkeninfection, die letal endigte, konnte ich in mehreren Fällen neben zahlreichen Streptokokkenketten bis zuletzt Stäbchen in grosser Zahl nachweisen. Auch Heynemann berichtet über derartige Fälle, wo Kokken und Stäbchen in gleicher Anzahl vorhanden waren und der Fall tödtlich endigte. 
Bei vielen weniger schweren Infectionen, die nur mit kürzer oder länger dauernden Fiebersteigerungen einhergingen, aber doch als inficirte Fälle anzusehen waren, war es überhaupt das gewöhnliche Bild, dass man neben den zahlreichen Leukocyten eine Unzahl Stäbchen und zumeist auch Kokken fand. Dieselben liegen extra- und intracellulär und die Leukocyten sind oft dicht mit ihnen vollgepfropft.

In den zahlreichen Fällen, in denen man nur Diplokokken im Ausstrich findet, ergiebt sich ferner die sehr wichtige Frage, um welche Art von Kokken es sich dabei handelt, ob um Streptokokken, Staphylokokken, Pneumokokken, anaerobe Kokken, Gonokokken oder andere. Diese Frage kann dann immer nur durch die Cultur entschieden werden oder, soweit es die Gonokokken betrifft, durch Anfertigung des Gram-Präparates. Aus der Kürze oder der Länge der Streptokokkenketten auf den Grad der. Virulenz zo schliessen, erwies sich mir ebenfalls als nicht zulässig.

Ich konnte in nur 20 pCt. der Fälle aus dem Objectträgerausstrich die Wahrscheinlichkeitsdiagnose stellen, die dann nachher durch die culturelle Untersuchung bestätigt wurde und mit dem klinischen Verlaufe übereinstimmte.

In $47 \mathrm{pCt}$. der Fälle versagte der Objectträgerausstrich gegenüber der Culturmethode vollkommen, in $6 \mathrm{pCt}$. leistete das Ausstrichpräparat zur Diagnose der Gonorrhoe gute Dienste, in $27 \mathrm{pCt}$. konnte man im Secret Streptokokken vermuthen.

Ebenso wenig konnte man aus der Phagocytose, aus der Lagerung oder aus der Menge der Streptokokken irgend einen sicheren Rückschlnss auf die Schwere der Infection machen. Und je mehr ich mich bemühte, feststehende Normen hierfür aufzustellen, desto häufiger wurde ich durch das bakteriologische Culturergebniss und den klinischen Verlaut ențäuscht.

Ich kann daher Heynemann nur beipflichten, der die Unzuverlässigkeit des Objectträgerausstriches betont, von dem er in vielen Fällen nur irre geführt wurde.

Niemals ist es mir vorgekommen, dass ich Streptokokken im Ausstrichpräparat nachgewiesen hätte, die ich nachher nicht auch culturell gefunden hätte.

Zangemeister, der über zwei derartige Fälle berichtet, führt selbst nachher an, dass er damals noch nicht die Blutagargussplatte als Culturverfahren benutzt hat. Im Uebrigen kommt auch Zangemeister auf Grund seiner Ergebnisse: In 22 pCt. der. Fälle 
296 Goldschmidt, Methoden zur Untersuchung des Lochialsecretes u.s.w.

waren die Streptokokken mit dem Mikroskop nachweisbar, in $56 \mathrm{pCt}$. der Fälle konnte er sie vermuthen und in $15 \mathrm{pCt}$. der Fälle sie überbaupt nicht nachweisen, zu dem Schluss, dass diese Art der Diagnose auch nicht den bescheidensten Ansprüchen der bakteriologischen Diagnostik entspricht.

Damit glaube ich die ausserordentlich geringe Bedeutung, die dem Ausstrichpräparat für die Diagnose der Streptokokkeninfection insonderheit aus den Scheidenlochien zukommt, genügend dargelegt zu haben.

Und doch hat das Ausstrichpräparat zweifellos bei fieberhaften Erkrankungen im Wochenbett einen grossen Werth, nämlich dann, wenn es sich um die Erkennung einer Gonokokkeninfection handelt. Hier leistet es ganz vorzügliche Dienste und ist wegen seiner bequemen Anfertigung durch Secretentnahme mit der Platinöse aus dem vordersten Theil der Scheide auch dem Praktiker sehr zu empfehlen.

Was die Frage der Verwendung flüssiger Nährböden anbelangt, so muss man hier 2 wesentliche Punkte unterscheiden:

1. Ob es mit Hilfe derselben möglich ist, Streptokokken nachzuweisen, wo dieses mit anderen Culturmethoden nicht mehr gelingt und

2. Inwieweit der flüssige Nährboden geeignet ist, die Diagnose und in gewissem Sinne auch die Prognose des Puerperalfiebers zu stellen.

Ueber den zuerst angeführten Punkt besteht insofern eine Verschiedenheit in den Auffassungen, als eine Reihe von Untersuchern die flüssigen Nährböden, vor Allem die Bouillon, schwach alkalisch oder mit Traubenzucker oder Ascites und anderem Zusatz für ein ausserordentlich feines Reagenz zum Nachweis des Streptococcus pyogenes halten, das den festen Nährböden überlegen sei.

Durch die Untersuchungen von Walthard, Bumm, Sigwart, Schenk, Scheib u. A. wurde unter Benutzung der flüssigen Nährböden denn auch zuerst die Lehre, dass der Streptococcus pyogenes im Scheidensecret Hochschwangerer nicht vorkomme, angegriffen und anscheinend widerlegt. Mit Hilfe der Bouillon fand man weiterhin zuerst, dass im Lochialsecret nichtfiebernder Wöchnerinnen in einer bis dahin ungeabnten Häufigkeit Streptokokken zu finden sind.

Menge und Krönig haben jedoch durch sehr gründliche Versuchsreihen bereits den Beweis erbracht, dass man durch Verwen- 
dung von Agar als Nährboden bei der Aussaat von Scheidensecret den Streptococcus pyogenes des Puerperalfiebers resp. sein Fehlen ebenso sicher nachzuweisen vermag, wie bei der Verwendung von Bouillon.

In einer neueren Publication gibt Krönig zwar zu; dass für die Streptokokken des Scheidensecretes, die natürlich -nicht ohne Weiteres mit dem Streptococcus pyogenes puerperalis identisch sind, die Traubenzuckerbouillon zwar einen besseren Nährboden darstellte als das schwach alkalisch reagirende Agar, während er diesen Unterschied in der Qualität des Nährbodens für den aus dem Lochialsecret der inficirten Wöchnerin rein gezüchteten Streptococcus pyogenes puerperalis nicht anerkennt; er glaubt vielmehr, dass die Traubenzuckerbouillon sich besonders für den Nachweis der Streptokokken eignet, welche saprophytisch im Secret leben.

In dieser Richtung müssen daher erst noch weitere Untersuchungen Klarheit schaffen.

Wesentlich anders gestaltet sich die Frage, ob die Bouillon geeignet ist, die Diagnose und in gewissen Fällen auch die Prognose des Puerperalfiebers zu fördern. Ursprünglich ging man hierbei ron der Ansicht aus, dass eine Eintheilung der Streptokokken nach ihrem Verhalten zur Bouilloncultur möglich sei. Man nahm an, dass der Streptococcus brevis, der wenig virulent sei, die Bouillon trübe, währẹnd der hochpathogene Streptococcus longus, der mit flockigem, schleimigem Bodensatz wachse, dieselbe klar lasse. Auch suchte man nach der Art des Wachthums und der verschiedensten Veränderungen der Bouillon immer wieder nach makroskopisch schon sichtbaren Unterschieden in der Eintheilung pathogener und nichtpathogener Streptokokken. Alle diese Bemühungen blieben indes vergeblich. Ebenso wenig gelang es nachzuweisen, dass der frisch gezüchtete typisch hämolysirende Streptococcus, der in schwach alkalischer Bouillon zumeist mit flockigem Bodensatz wächst, dieselbe immer klar lässt, während der nichthämolytische Streptococcus dieselbe diffus trübt.

Sigwart, der ein eifriger Fürsprecher der flüssigen Nährböden ist, glaubt indes in der That, aus der primären Cultur einen Unterschied zwischen virulenten und avirulenten Streptokokken machen zu können. Er fasst seine Ansicht dahin zusammen, dass er sagt: Liegt eine Infection mit Streptokokken vor, so wird die mit Lochialsecret beschickte Bouillon eine Reincultur ergeben. Liegt keine Infection vor, so wird die Bouillon entsprechend den verschieden- 
298 Goldschmidt, Methoden zur Untersuchung des Lochialsecretes u.s.w.

artigen Keimen im Lochialsecret meist eine Mischeultur ergeben, in der allerdings zahlreiche Streptokokken gewachsen sind. Eine aus Lochialsecret direct gewonnene Bouillonreincultur zeigt uns in der Regel an, dass lediglich der Streptococcus das bakteriologische Bild beherrscht und dies ist nur der Fall bei Streptokokkeninfectionen, nicht aber bei Anwesenheit des Streptococcus neben anderen Keimen.

Diese Behauptungen Sigwart's fand ich durch meine Untersuchungen nicht bestätigt. Ich konnte bei 75 fiebernden Wöchnerinnen mit der Bouilloncultur $56 \mathrm{mal}$, das heisst in circa 75 pCt. der Fälle Streptokokken nachweisen. Davon war in 24 Fällen die Bouillon rein, in den 32 übrigen Fällen unrein. Die Reinheit der Cultur entsprach hierbei durchaus nicht immer einer schweren Infection und unter den 32 Fällen, in denen eine Mischcultur mit Streptokokken in der Bouillon aufging, waren eine grosse Anzahl schwerer Infectionen.

Bei weiteren 50 nichtfiebernden Wöchnerinnen waren $31 \mathrm{Mal}$, also in $62 \mathrm{pCt}$. der Fälle Streptokokken nachweisbar, $9 \mathrm{Mal}$ war die Cultur rein, 22 Mal unrein. Auch dieser Befund hei fieberfreien Wöchnerinnen spricht gegen die Unhaltbarkeit der von Sigwart aufgestellten Behauptung.

Klarheit oder Trübung, Reinheit oder Unreinheit der Cultur, Kürze oder Länge der Ketten gestattete mir keinen sicheren Rückschluss zu diagnostischen Zwecken, oder auf die Schwere einer eventuell bestehenden Infection.

Besonders hervorheben möchte ich, dass unter den oben angeführten Fällen bei fiebernden und leichtfiebernden Wöchnerinnen vicle sind, bei denen in der Bouillon cine Reincultur von Streptokokken aufging, während sich auf den festen Nährböden nur ganz vereinzelte Keime von Streptokokken fanden. Hier hatten offenbar die wenigen eingesäten Streptokokken in der Bouillon in Folge der günstigen Ernährungsbedingung alle anderen Keime überwuchert, eine Thatsache, mit der man bei Aussaat auf. flüssige Nährböden immer rechnen muss.

Von anderen Untersuchern möchte ich nur die Resultate Zangemeister's erwähnen. Er fand bei fiebernden Wöchnerinnen die Bouilloncultur in 68 pCt. der Fälle rein, in 31 pCt. unrein und erklärt die auffallende Häufigkeit der Reinheit der Bouilloncultur damit, dass die virulenten Keime, die zur Infection führen, die anderen überwuchern und untergehen lassen. 
Sachs, der wie andere Untersucher keinerlei Parallelismen zwischen dem Nachweis der Streptokokken in der Bouillon und der Schwere der Krankheitsbilder fand, gab es bald auf, diese Frage systematisch weiter zu prüfen.

Der allergrösste Nachtheil aber, den die Bouillon hat, besteht darin, dass sie über die Zahl der eingesäten Keime nicht.im geringsten Auskunft giebt und dass Verunreinigungen bei ihr nicht erkannt werden können und so mitunter zu den unangenehmsten Trugschlüssen führt. Ich kann mich daher auf Grund meiner Untersuchungen nur denjenigen Forsehern anschliessen, die die Bouillon als alleiniges Material der Aussaat zur Diagnose des Puierperalfiebers als völlig ungeeignet ansehen.

Die Vorzüge der festen Nährböden an und für sich bestehen in der Hauptsache darin, dass man aus einem Keimgemisch, wie es das Genitalsecret ja auch zumeist zeigt, die einzelnen Arten und Colonien isoliren kann. Sie gewähren die Mögliehkeit, über das Verhältniss der einzelnen Keime zueinander und ihrer Menge Schlüsse zu ziehen.

Von vornherein stehen sich nun gegenüber der einfache Agar schwach alkaliseher Natur, sauer oder mit Traubenzucker, Glyzerin und anderem Zusatz versehen und der Blutagar, wie ihn Schottmüller zuerst in die Bakteriologie eingeführt hat. Durch eine grosse Reihe von Untersuchungen wurde dabei festgestellt, dass unter dem einfachen Agar zur Untersuchung des Lochialsecretes sich am besten der schwach alkalische Agar eignet, vor Allem soweit es den Streptokokkennachweis anbelangt, dass aber auch die anderen Keime, die ausserdem als pathogene im Lochialsecret in erster Reihe in Betracht kommen, auf ihm ein gutes Fortkommen finden.

Deshalb hält Krönig auch heute noch an der Verwendung der schwach alkalischen Agargussplatte fest.

Er zieht es vor, zunächst kleine Mengen Secret von etwa drei Platinösen auf schwach alkalischem Agar auszusäen und erst, wenn Streptokokken hierbei auf der Gussplatte in grösserer Menge aufgehen, sie auf ihre Hämolyse zu prüfen, um die Wahrscheinlichkeitsprognose zu stellen.

Zu diesem Verfahren Krönig's und Pankow's ist zu sagen, dass die Differenzen, die sich in der Feinheit der schwach alkalischen Gussplatte und der Blutagargussplatte ergeben, allerdings be- 
stehen, wenn sie auch nicht bedeutend sind. Ich habe nach sorgfältiger, jedesmal auch mikroskopischer Durchmusterung der Agarplatten dafür folgende Zahlen gefunden: Bei fiebernden Wöchnerinnen waren Streptokokken auf der schwach alkalischen Agarplatte in 43 pCt. der Fälle nachweisbar, auf der Blutagargussplatte in 48 pCt. der Fälle; bei nicht fiebernden Wöchnerinnen betrug das Verhältniss $17: 21 \mathrm{pCt}$.

Es wäre nun von Werth, wenn es sich in denjenigen Fällen, in denen die Agarplatte versagt, dagegen die Blutplatte einen positiven Befund zeigt, der Streptokokkenbefund bedeutungslos wäre. Es würden dadurch eine Reihe von Fällen mit positivem Streptokokkenbefund als belanglos ausscheiden. Dem ist aber nicht so. Andererseits fand ich mitunter, dass die Schottmüller'sche Blutagarplatte eher und sicherer das Vorhandensein von Streptokokken anzeigte als die schwach alkalische, auch in solchen Fällen, die nachher mit länger dauernden Fiebersteigerungen einhergingen.

Dass der schwach alkalische Agar an Empfindlichkeit der Blutagargussplatte nachsteht, konnte ich immer wieder bei meinen Untersuchungen feststellen.

Ich bin daher auf Grund meiner Untersuchungen zu der Ueberzengung gekommen, dass die beste Methode der Aussaat des Lochialsecretes zum Nachweis von Streptokokken, die uns heute zur Verfügung steht, die auf Blutagar ist. Sie hat vor der schwach alkalischen Agarplatte den Vortheil, dass sie ein feineres Reagens ist. Sie ist ferner praktischer, weil sie ohne Weiteres, auch schon ohne Zuhülfenahme des Mikroskops, sofort die wichtigsten Keime in charakteristischer, nicht $\mathrm{zu}$ übersehender Weise anzeigt. So. zeigt sie in scharf differenzirender Weise den Streptococcus haemolyticus und den Streptococeus viridans an, ferner den Staphylococcus haemolyticus und nichthaemolyticus, das Bacterium coli commune und haemolyticum, den Pneumococcus, Gonococcus u. A. Und wenn man wirklich auf das hämolytische Verhalten der Streptokokken besonderen Werth legen will, so ist es doch nur eine doppelte Arbeit, die man sich macht, wenn man zunächst auf Agar aussät und dann erst die Colonien auf die Blutplatte überträgt, um die hämolytischen Fähigkeiten der Keime festzustellen, wodurch ausserdem auch das Ergebniss der Untersuchungen um viele Stunden hinausgeschoben wird.

So zweifellos die Blutagargussplatte für den Nachweis der Streptokokken zur Zeit den einwandfreiesten und besten Cultur- 
boden darstellt, so sicher ist es; dass sie das Vorhandensein von Streptokokken in einer überaus grossen Anzahl von Fällen anzeigt, wo ejgentlich von einer Infection im klinischen Sinn auch nicht im geringsten die Rede sein kann. Es beweisen das die vielen Fälle, wo ich bei fieberfreien Wöchnerinnen durch eine Reihe von Tagen hindurch die Blutplatte mit Streptokokken übersät fand, ohne dass das Wochenbett nur die geringsten Störungen erlitten hätte.

Nun glaubte Zangemeister und ganz besonders auch Sachs, diese Fälle ausscheiden zu können, wenn man das Lochialsecret nur oberflächlich auf eine Blutagarplatte verimpfte. Zeigte es sich dabei, dass die Streptokokken die Fähigkeit besässen, alle anderen Keime zu überwuchern, so war es für Sachs sicher, dass es sich um eine schwere Infection mit Streptokokken handeite.

So lange ich fiebernde Wöchnerinnen untersuchte, schien sich diese Ansicht auch mir zu bestätigen. Während die Blutagargussplatte in 48 pCt. der Fälle Streptokokken anzeigte, geschah dieses bei dem Oberfächenausstrich nur in 33 pCt. und diese Fälle waren fast ausnahmslos die schweren, zum Theil letal verlaufenden. Die breite, streifenförmige, glänzende Hämolyse im Verlauf des Impfstriches war für mich zum Zeichen einer vorhandenen schweren Infection geworden, und so lange ich nur fiebernde Wöchnerinnen untersuchte, fühlte ich mich berechtigt, die Temperatursteigerung auf das Vorhandensein dieser Streptokokken zurückzuführen. In zahlreichen anderen Fällen, wo im Lochialsecret neben Streptokokken noch andere Keime vorhanden waren und wo dann auch auf der Blutagargussplatte vereinzelte Streptokokkencolonien aufgingen, konnte ich wiederholt beobachten, dass beim Impfstrich auf der Blutplatte diese wenigen Streptokokkencolonien von den anderen zahlreicher vorhandenen Keimen thatsächlich äberwuchert wurden.

Als ich aber anfing, systematisch 50 fieberfreie Wöchnerinnen zu untersuchen, erwies sich mir auch dieses Rriterium des breiten hämolytischen Striches nicht mehr als einwandsfrei. Ich suchte mir bei meinen Untersuchungen mit Vorliebe solche Wöchnerinnen aus, bei denen es sich um manuelle Eingriffe bei der Entbindung gehandelt hatte, um Steisslagen mit Armlösung, um Episiotomien, um Dammnähte, und bei denen dennoch das Wochenbett ungestört verlief. Ich war überrascht, in $22 \mathrm{pCt}$. dieser Fälle den schönsten von Streptokokken herrührenden hämolytischen Impfstrich auf der Blutplatte zu finden, während die Temperatur dieser Wöchnerinnen nie-

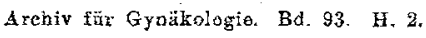


mals $38^{\circ} \mathrm{C}$. erreichte und sie nach $8-10$ Tagen wohlbehalten die Klinik verlassen konnten.

Will man überhaupt allemal die Streptokokken, die Hämolyse bewirken, als besonders virulent, oder zum mindesten als mit besonderer Wachsthumsenergie begabt betrachten, so muss hier auf die grosse Zahl von Wöchnerinnen hingewiesen werden, die mit einer Unzahl solcher hämolytischer Streptokokken in der Vagina nicht die geringsten klinischen Erscheinungen bekommen. Krönig ist der Meinung, dass man auch alle diese Fälle als inficirt betrachten und sie als Endometritis streptococcica bezeichnen müsse, und ich kann mich dieser Meinung nur durchaus anschliessen. Dass dadurch leicht Missverständnisse zu Tage treten können im Gegensatz zu der bisherigen Anschaung der meisten Autoren liegt auf der Hand. Wie sçhwer es hält, zu unterscheiden, ob das Fieber wirklich durch die in der Scheide gefundenen Streptokokkenkeime hervorgerufen wird oder nicht, erhellt aus der von mir bereits oben angeführten Thatsache, dass man gerade in solchen Fällen, wo manuelle Eingriffe vorhergegangen sind, die hämolytischen Streptokokken in grosser Menge auch bei Nichtfiebernden findet. Auch beim Oberflächenausstrich wachsen sie in solchen Fällen oft mit so ausgesprochener Hämolyse, dass dieselbe als zuverlässiger Maassstab für das Bestehen einer schweren puerperalen Erkrankung nicht gelten kann.

Ueberhaupt muss man in der Verwerthung des bakteriologischen und bakterioskopischen Befundes mit der allergrössten Vorsicht zu Werke gehen.

Namentlich für die in der Praxis stehenden Geburtshelfer und besonders für die Hebammen hat diese Frage eine ganz eminente Bedeutung, auf die ich hier kurz hinweisen möchte. Jedenfalls müsste auf Grund der Thatsache, dass auch in der Scheide nicht fiebernder Wöchnerinnen Streptokokken gefunden werden, die für die betreffende Wöchnerin nicht aggressiv sind, bei Uebertragung auf andere Wöchnerinnen es aber sicherlich sein oder werden können, eine Aenderung in den jetzt für die Hebammen geltenden Bestimmungen eintreten.

Nachdem die so bisher geübte Methode zur Untersuchung des Lochialsecretes auf Streptokokken mit ihren im Vorstehenden angeführten Vor- und Nachtheilen wohl zur Diagnose einer Infection mit Streptokokken, aber nur in bedingtem Sinne zur Stellung der Diagnose und Prognose des Puerperalfiebers Geltung beanspruchen 
Goldschmidt, Methoden zur Untersuchung des Lochialsecretes u.s.w. 303

konnte, lag es auf der Hand, nach weiteren Merkmalen zwischen dem pathogenen penetrirenden Streptococens und dem nur saprophytischen nicht aggressiven zu fahnden.

Fromme glaubte nun ein solches Unterscheidungsmerkmal in seinem Blutschwammverfahren gefunden zu haben; aber die Nachuntersuchungen sowohl an unserer Klinik durch Schottelius, als auch durch Andere (Bondy etc.) haben ergeben, dass sich auch die Blutschwammpassage praktisch zur Prognosestellung nicht verwerthen lässt.

Ein weiteres Verfahren, das nach Fromme ebenfalls und zwar noch besser als das Blutschwammverfahren die Möglichkeit einer exacten Unterscheidung zwischen hämolytischen nichtpathogenen Eigenstreptokokken und pathogenen hämolytischen Fremdstreptokokken gewähren soll, bildet sein Lecithinemulsionsverfahren. Dasselbe soll darauf beruhen, dass saprophytische Streptokokken in einer Mischung von Bouillon mit einer 2proc. Lecithinemulsion ein quantitativ anderes. Wachsthum zeigen, als parasitäre. Bei einer sich gleichbleibenden Tropfenzahl, z. B. von 13 Tropfen Lecithin + Bouillon sollen die pathogenen Stärnme erst bei einem höheren Gehalt des Nährbodens in Bouillon wachsen, also etwa beim Verhäitniss $10 \mathrm{~B}: 3 \mathrm{~L}$, während die saprophytischen bereits bei einer viel niedrigeren Concentration gedeiben.

Dieses Verfahren, welches wie das Blutsehwammverfahren ebenfalls sehr umständlich ist, will Fromme dadurch vereinfachen, dass er die Bouilloncultur umgeht. Er will das auf Streptokokken zu untersuchende Lochialsecret gleich in die verschiedenen Bouillonlecithinmischungen impfen, ohne dass dadurch das Resultat beeinflusst werden soll.

Der Versuch gestaltet sich dementsprechend wie folgt:

Beimpfung von 5 Röhrchen in der Concentration von

1. $7 \mathrm{~B}: 6 \mathrm{~L}$
2. $8 \mathrm{~B}: 5 \mathrm{~L}$
3. $9 \mathrm{~B}: 4 \mathrm{~L}$
4. $10 \mathrm{~B}: 3 \mathrm{~L}$
5. $11 \mathrm{~B}: 2 \mathrm{~L}$

mit je einer Normalöse des aus der Scheide zu entnehmenden Lochialsecretes; 12stündige Bebrütung; Giessen der Blutplatte in der ü̈blichen Weise unter Zusatz des bebrüteten Gemisehes. Controle der Platte nach 12 stündigem Aufenthalt im Brutofen. 
304 Goldschmidt, Methoden zur Untersuchung des Lochialsecretes u.s.w.

Erscheinen, so führt Fromme aus, auf den gegossenen fünf Platten auf jeder reichlich hämolytische Streptokokken, so sind es saprophytische Streptokokken. Bleiben die Platten 1--3 oder $1-4$ oder alle steril, wuchsen auf dem Blutagarausstrich aber hämolytische Streptokokken, so handelt es sich sicherlich um pathogene hämolytische Fremdstreptokokken und es liegt eine puerperale Infection bei dem betreffenden Fall vor.

Diese Angaben, die Fromme schon dem Praktiker als unumstössliche Thatsache hinzustellen für gut fand, bedürfte natürlich sehr der Nachuntersuchung auf ihre Richtigkeit. Schon auf der Versammlung der mittelrheinischen Gesellschaft in Frankfurt konnte ich auf Grund meiner Nachprüfung im Anschluss an die Ausführungen Traugott's aus der Walthard'schen Klinik mich dem ablehnenden Standpunkt Walthard's und Traugott's völlig anschliessen.

Im Ganzen stellte ich Versuche mit 20 hämolytischen Streptokokkenstämmen an. 6 Stämme waren aus dem Vaginalsecret schwer fiebernder Wöchnerinnen gezüchtet, 1 Stamm aus dem parametranen Eiter bei schwerer Infection mit Streptokokken und 13 Stämme aus dem Lochialsecret nicht oder nur ganz vorübergehend fiebernder Wöchnerinnen.

Bei den 2 letal endigenden Fällen erwies sich der gezüchtete Streptococcus im Fromme'schen Lecithinversuch im einen Falle parasitär, im anderen dagegen saprophytär.

Der Stamm, der aus dem Eiter einer schweren, in wenig Tagen sich entwickelnden und über dem Ligamentum Poupartii durchgebrochenen parametranen Abscess gezüchtet wurde, erwies sich ebenfalls als nicht pathogen.

Von den weiteren 4 Stämmen, die klinisch schwere Erscheinungen machten, war bei 3 ausgesprochene Wachsthumshemmung zu constatiren, während sie im 4. Falle fehlte. Alle 4 Fälle genasen.

Unter den 13 Stämmen, die von nicht oder nur leicht fiebernden Wöchnerinnen stammten, waren 5, bei denen man im Frommeschen Versuch auf eine hohe Virulenz der Streptokokken hätte schliessen müssen, und doch traten keinerlei Erscheinungen im Wochenbett auf. Die übrigen 8 Stämme verhielten sich wie saprophytäre Streptokokken.

Nach diesen Resultaten kann ich nur meinen bereits früher geäusserten Standpunkt wiederholen, dass von einer einwandfreien 
Unterscheidung der virulenten Stämme mit hohem Penetrationsvermögen und solchen mit rein saprophytären Eigenschaften mittelst des Fromme'schen Lecithinversuches nicht die Rede sein kann. In Folge dessen habe ich auch die weitere Vereinfachung des Verfahrens mit der sofortigen Einsaat des Lochialsecretes in die Bouillon-Lecithinemulsion nicht weiter verfolgt, da ich deren Ergebnisse natürlich für ebensó unsicher hielt, wie die mit den rein gezüchteten Streptokokkenstämmen.

So ist das Problem der Differencirung von virulenten und avirulenten Streptokokken trotz der zahlreichen Bestrebungen und der wiederbolten Versuche, und trotzdem besonders Fromme wiederholt glaubte, den Schlüssel dazu gefunden zu haben, völlig ungelöst.

Ich bin der Ansicht, dass eine Stellung dieser Diagnose und damit der Prognose des Puerperalfiebers auch stets scheitern wird, solange man sie nur ans dem rein bakteriologisehen Befund zu stellen sucht. Ich bin vielmehr der Meinung, dass, worauf auch Opitz und Menge auf dem letzten Gynäkologencongress in Strassburg erst nachdrücklich wieder hingewiesen haben, man den Factor Mensch, das Individuum mit seiner verschiedenen Resistenz, bisher viel zu sehr ausser Acht gelassen hat. Und auch die Versuche von Sachs, für alle nur in Betracht kommenden Factoren zur kritischen Beurtheilung des Falles eine annähernd zuverlässige mathematische Schätzungsformel aufzustellen, sind zu sehr individuellen Schwankungen unterworfen, als dass sie allgemeine Gültigkeit beanspruchen könnten.

Solange wir aber nichts Besseres haben, wird man versuchen müssen, aus der bakteriologischen Untersuchung des Lochialsecretes mit Berücksichtigung der mechanischen Verhältnisse im Genitaltractus, der Schutz- und Abwehrkräfte des Organismus, der morphologischen und bakteriologischen Untersuchung des Blutes und des gesammten klinisehen Bildes zu einem möglichst zuverlässigen Resultat zu kommen.

Soweit es sich dabei um die Untersuchung auf aerobe Streptokokken handelt, wird man sich zu ihrem Nachweis der im Torstehenden geschilderten zweckmässigsten Methoden zu bedienen haben, die man dazu noch am besten nebeneinander ausführt, um so am sichersten Fehlerquellen auszuschliessen.

Für den Praktiker dürfte nach wie vor das klinische Bild zur Beurtheilung des Falles einzig und allein maassgebend bleiben. 
Mit ganz besonderem Nachdruck möchte ich schliesslich die Aufmerksamkeit aber für die Untersuchung des Lochialsecretes auch auf die anaeroben Bakterien lenken, die in der Pathologie des Wochenbettes eine bisher nicht genügend gewürdigte Rolle spielen.

Es liegt sicherlich zu einem beträchtlichen Theil an der etwas umständlichen und mühsamen Züchtung dieser Keime, dass ihnen bisher noch nicht die Stellung eingeräumt ist, die ihnen gebührt.

Menge und Krönig haben zuerst 1895 und dann in wiederholten Publicationen auf die Bedeutung eines obligat anaeroben Streptococcus aufmerksam gemacht, den sie im Genitaltractus der Frau fanden und dem sie auf Grund ihrer klinischen Erfahrung schon damals eine pathogene Bedeutung zumaassen.

Während die meisten Untersucher des Lochialsecretes diese Bakterien gar nicht oder kaum berücksichtigten, hat vor Kurzem Schottmüller in einer ausführlichen Arbeit gerade die pathogene Bedeutung des Streptococcus anaerobicus oder putridus, wie er ihn nennt, eingehend dargelegt und über die Häufigkeit seines Vorkommens und seine Bedeutung namentlich bei putriden Processen auch ausserhalb des Genitaltractus sehr werthvolle Mittheilungen gemacht.

Die Züchtung des Streptococcus anaerobicas gesehieht am besten in der hohen Schichtcultur, wie ich sie Eingangs beschrieb, zumal die Pyrogallussäure für Plattenculturen sich nicht durchweg bewährt hat. Da für die Pathogenität des anaeroben Streptococcus sein Vorkommen im Blut von besonderer Bedeutung ist, so hat Schottmüller für diesen Nachweis recht brauchbare und zum Theil bequeme Verfahren angegeben. Er bringt das zu untersuchende Blut in Bouillon und ohne weitere Behandlung in den Brutschrank. Unter scheinbar aeroben Bedingungen entwickeln sich dann hier innerhalb einiger Tage Streptokokken, die man weiterhin als streng anaerobe erkennt. Diese Thatsache ist so zu erklären, dass in Folge des zusammenballenden Blutes ein vollkommener Abschluss der in der Tiefe befindlichen Bakterien vom Sauerstoff zu Stande kommt. Leider ist aber diese Blutbouilloncultur nicht immer zuverlässig, was ich selbst wiederholt störend empfand.

Es ist daher ein von Schottmüller angegebenes neues Verfahren, das Blut zur Schüttelcultur in Zuckeragar zu verwenden, wodurch man gute und brauchbare Resultate erhält, besonders lebhaft zu begrüssen. Da dieses Verfahren noch zu wenig bekannt sein dürfte, so führe ich dasselbe nach den Angaben Schott- 
müller's hier an. "Verwendet werden dazu Cylinder von $20 \mathrm{~cm}$ Länge, $5 \mathrm{~cm}$ Durchmesser, die mit ca. $25 \mathrm{ccm}$ Zuckkeragar gefüllt und steril bereit gehalten werden. Die Anlegung der Blutcultur gestaltet sich nun höchst einfach. Mit der trocken im Glascylinder sterilisirten Luer'schen Spritze von $20 \mathrm{cem}$. Werden aus der mit Gummiband gestauten Armvene (Vena mediana basilica) nach vorheriger Reinigung mit Aether $10-20 \mathrm{ccm}$ Blut dem Kranken entnommen und sofort in den Cylinder mit dem Zuckeragar, der zuvor verflüssigt und sorgfältig auf $45^{\circ}$ abgekühlt ist, hineingespritzt. Schaum- und Luftblasenbildung ist dabei unbedingt zu vermeiden. Nachdem Blut und Agar durch Sehwenken des Cylinders innig vermischt sind, wird die Flüssigkeit in kaltem Wasser schnell zum Erstarren gebracht und die Cultur in den Brutschrank gestellt. In der bekannten Weise werden dann die in der Agarsäule vorhandenen Colonien "aufgeschlossen".

Ueber die Art des Wachsthums des anaeroben Streptococcus verweise ich auf die eingehenden Schilderungen von Menge und Krönig.

Es soll hier aber noch besonders hervorgehoben werden, dass neben dem von Schottmüller so häufig nachgewiesenen Streptococcus anaerobus oder putridus es auch noch andere bereits von Menge und Krönig differencirte obligat anaerobe Streptokokken giebt, die nicht die Eigenschaft haben, übelriechende Zersetzung herbeizuführen.

Nachgewiesen wurde der Streptococcus anaerobicus bereits von Menge und Krönig

1. in der Scheide Hochschwangerer,

2. im Lochialsecret des Uterus,

3. im Fruchtwasser,

4. in intraperitonealen abgekapselten Abscessen,

5. in freien entzündlichen Bauchhöhlenexsudaten bei diffuser Peritonitis.

Diese Autoren heben ferner hervor, dass neben dem ausschliesslichen Vorkommen von facultativ anaeroben auch eine Symbiose dieser beiden Arten bei jauchiger Parametritis und im Lochialsecret des Uterus von ihnen beobachtet wurde. Durch Untersuchungen an der Leiche konnten sie schon damals auf die sicher pathogene Bedeutung des anaeroben Streptococcus hinweisen. 
308 Goldschmidt, Methoden zur Untersuchung des Lochialsecretes u.s.w.

Diesen viel angefeindeten und bezweifelten Behauptungen Menge's und Krönig's ist durch die Arbeit Schottmüller's eine glänzende Rechtfertigung und eine ganz ungeahnte Erweiterung zu Theil geworden. An der Hand von 25 Krankengeschichten weist Schottmüller nach, dass der in dem erkrankten Organ gefundene obligat anaerobe Streptococcus als streng pathogen anzusehen ist. Derselbe war $14 \mathrm{mal}$ mit anderen Keimen vergesellschaftet, wo seine ätiologische Mitwirkung bei der Erkrankung nicht in Abrede zu stellen war. In weiteren 11 Fällen hat er sicher ganz allein das Krankheitsbild verursacht. Bei folgenden Erkrankungen konnte Schottmüller den Streptococcus anaerobicus putridus nachweisen: Otitis medja, Meningitis, Cystopyelitis, Abscessus pulmonum, Gangraena pulmonum(Tuberculosis pulmonum), Empyema pleurae, Endometritis putrida, Salpingitis, Parametritis, Peritonitis, perimetritischer Abscess, Sepsis mit Thrombophlebitis.

Dabei ist der anaerobe Streptococcus vergesellschaftet mit den verschiedensten Keimen, aber auch ein reiner Fall von Alleininfection wird von Schottmüller beschrieben.

Bemerkenswerth ist, dass der Streptococcus anaerobicus allein

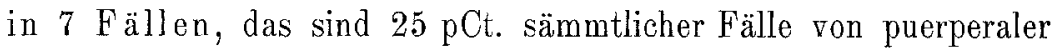
Sepsis, die bei Schottmüller zum Exitus führten, der eigentliche und einzige Erreger war. Wenn auch diese hauptsächlich für Aborte und zum Theil criminelle Aborte in Betracht kommenden Zahlen nicht ohne weiteres auf die puerperale Sepsis nach normalem Partus zu übertragen sind, so dürfte es doch an der Zeit sein, weit mehr als bisher diesem Keim auch in solchen Fällen Aufmerksamkeit zu schenken.

Ueber das Vorkommen des anaeroben Streptococcus im gesunden Organismus hat Schottmüller keine Erfahrung. Ich kann dieselben durch die bei meinen Lochialsecretuntersuchungen erzielten Ergebnisse ergänzen.

Bekanntlich wiesen schon Krönig und Menge als Erste den anaeroben Streptococcus auch unter normalen Verbältnissen in der Scheide bei Schwangeren und Nichtschwangeren nach.

Bei 125 Lochialsecretuntersuchungen hatte ich folgendes Ergebniss:

Bei 50 nichtfiebernden Wöchnerinnen fand ich 7 mal anaerobe Streptokokken zum Theil in Symbiose mit anderen Bakterien.

In 2 Fällen war es bemerkenswerth, dass, worauf schon Krönig aufmerksam machte, man auf dem Objectträgerausstrich 
Goldschmidt, Methoden zur Untersuchung des Lochialsecretes u.s.w. 309

eine grosse Anzahl von Kokken und Stäbchen fand, während aerobe auch nicht in einer einzigen Colonie auf den sämmtlichen Nährböden aufgingen. Die beiden hochgeschichteten Traubenzuckerculturen waren dagegen bis zur Sauerstoffgrenze durchsetzt mit zahllosen Colonien, die zunächst nur unscheinbar und klein, nach 3 mal 24 Stunden sich zu ansehnlichen, schon mit dem blossen Auge sichtbaren Colonien entwickelt hatten.

Sämmtliche ausgestochenen und weitergezüchteten Colonien erwiesen sich als einwandsfreie, typisch obligat-anaerobe Streptokokken. Charakteristisch war in den meisten dieser Fälle ein übelriechender stinkender Ausfluss. Fiebersteigerung war im ganzen Verlaufe des Wochenbettes nicht vorhanden.

Bei 75 fiebernden Wöchnerinnen waren $11 \mathrm{mal}$ anaerobe Streptokokken im Lochialsecret nachweisbar. Auch hier liessen in 3 Fällen die aeroben Plattenculturen völlig im Stich. Es gingen nur ganz vereinzelte Colonien von Bacterium coli commune und Staphylococeus pyogenes aureus auf, während die Schichtculturen den anaeroben Streptococcus in grosser Zahl aufwiesen. Auch in diesen Fällen, von denen keiner zum Exitus fübrte, bestand klinisch mehr oder weniger hohes remittirendes Fieber, dessen Dauer zwischen einigen Tagen und mehreren Wochen schwankte, und übelriechender, stinkender Ausfluss. In den 8 übrigen Fällen, wo die anaeroben Streptokokken vergesellschaftet waren mit anderen aeroben Keimen, war der Antheil, den sie an der Fiebersteigerung hatten, nicht mit Sicherheit zu bestimmen.

Den einwandfreien Beweis für die parasitären Eigenschaften der anaeroben Streptokokken habe ich in vivo überhaupt nicht erbringen können, da ich die Untersuchung des Blutes auf anaerobe Streptokokken nicht systematisch durchgeführt habe, und ich erst durch die Arbeit Schottmüller's, die nach Abschluss meiner Untersuchungen erschien, auf die zweckmässige Untersuchung des Blutes mit der Cylindercultur hingewiesen wurde.

In Folge der überraschenden Ergebnisse Schottmüller's mit diesem Verfahren wird man daher in Zukunft als unbedingtes Erforderniss verlangen müssen, dass nicht nur eine fortwährende Controle des Blutes auf aerobe sondern auch auf anaerobe Streptokokken vorgenommen wird, um so den Einbruch der Bakterien in die Blutbahn rechtzeitig zu erkennen und dementsprechend das therapeutische Handeln einzurichten.

Auf diese Weise wird sicherlich mancher bisher unklare Fall 
310 Goldschmidt, Methoden zur Untersuchung des Lochialsecretes n.s.w.

ätiologisch und bakteriologisch seine richtige Deutung finden. Ieh selbst habe einen Fall erlebt, in dem eine Patientin mehrere Wochen fast täglich einen oder mehrere langdauernde, schwere Schüttelfröste hatte mit hohem remittirendem Fieber und übelriechendem Ausfluss. Im Lochialseeret konnten anaerobe Streptokokken in grosser Zahl nachgewiesen werden, daneben vereinzelt Colonien von Staphylococcus pyogenes aureus. Trotzdem joh wiederholt während und unmittelbar nach dem Schüttelfrost jedesmal 5--6 Blutplatten gegossen hatte, blieben stets sämmtliche Platten steril. Anaerobe Blutculturen habe ich damals noch nicht angelegt. Ich zweifle aber nicht daran, dass, da es sich in diesem Falle wohl sicher um eine Infection mit dem anaeroben Streptococcus gehandelt hat, man dies mit der Cylindercultur wahrscheinlich hätte nachweisen können.

Weitere ausgedehnte Untersuchungen über das Vorkommen von anaeroben Bakterien und besonders von anaeroben Streptokokken werden sicherlich noch recht interessante Aufschlüsse über die Aetiologie des Puerperalfiebers bringen.

Wenn man bisher auch mit Krönig der Meinung war, dass im Allgemeinen die anaeroben Streptokokken klinisch nur geringe locale und allgemeine Erscheinungen machen, so haben die Untersuchungen Schottmüller's gezeigt, dass der Streptococcus putridus doch in einer erheblicheren Anzahl als man bisher annahm zur tödtlichen puerperalen Sepsis führen kann. Eine unbedingte Forderung für alle bakteriologischen Untersuchungen zur Klärung des Puerperalfiebers wird daher sein, dass neben der Untersuchung auf aerobe Bakterien auch stets eine solehe auf anaerobe durchgeführt wird, und dass dabei dem Nachweis des Streptococcus anaerobicus im Lochialsecret und im Blut eine grössere Aufmerksamkeit geschenkt werden muss, als dies bisher geschehen ist.

\section{Li t e ratur.}

1. Menge und Krönig, Die Bakteriologie des weiblichen Genitalcanals. Leipzig. Arthur Georgi.

2. Menge und Krönig, Die Wahl des Nährbodens bei dem culturellen Nachweise geringer Streptokokkenmengen. Centralbl. f. Gynäkol. 1900.

3. Menge und Krönig, Monatssehrift f. Geburtstülfe u. Gynäkologie. Bd. 9. S. 703. 
4. Schottmüller, Zur Bedeutung einiger Anaeroben in der Pathologie, insbesondere bei puerperalen Erkrankungen. Mittheil. a. d. Grenzgebiet. d. Med. u. Chir. 1910. 21. Bd. 3. Heft.

5. F. Fromme, Die Physiologie und Pathologie des Wochenbettes. Berlin. 1910. (S. Karger.)

6. Fromme, Ueber die Unterscheidung der hämolytischen virulenten von den bämolytischen nicht virulenten Streptokolkken. Centralbl, f. Gynäkol. 1908. S. 1213.

7. Fromme, Neue Untersuchungen über die Differenzirung der hämolytischen Streptokokken. Centralbl. f. Gynäkol. 1909. S. 1217.

8. Krönig und Pankow, Zur bakteriologiscben Diagnose des Puerperalfiebers. Centralbl. f. Gynäkol. 1909. S. 161.

9. Pankow, Zur bakteriologischen Diagnose des Puerperalfiebers. Centralbl. f. Gynäkol. 1909. S. 755 .

10. Sigwart, Zur bakteriologischen Diagnose des Puerperalfiebers. Centralbl. f. Gynäliol. 1909. S. 519.

11. Sigwart, Zar bakteriologischen Diagnose des Puerperalfiebers. Centralbl. f. Gynäkol. 1909. S. 1013.

12. Zangemeister, Verhandl. d. deutsch. Gesellsch. f. Gynäkol. 1909. Bd. 13. S. 313.

13. Opitz, Verhandl. d. deutsch. Gesellsch. f. Gynäkol. 1909. Bd. 13. S. 328.

14. Menge, Verhandl. d. deutsch. Gesellsch. f. Gyn. 1909. Bd. 13. S. 332.

15. Sachs, Verhandl. d. deutsch. Gesellsch. f. Gyn. 1909. Bd. 13. S. 395.

16. Bondy, Verhandl. d. deutsch. Gesellsch. f. Gyn. 1909. Bd. 13. S. 388.

17. Walthard und Traugottt, Zur Differenzirung von Streptokokkenstämmen mit und ohne Penetrationsvermögen. Monatsschr. f. Geburtsh. u. Gynäkol. 1910. Bd. 31. S. 777.

18. Goldschmidt, Discussion zu diesem Vortrag. Referat. Monatsschr, f. Geburtsh. u. Gynälkol. Bd. 31. S. 778.

19. Sachs, E., Bakteriologische Untersuchungen beim Kindbettfieber. Zeitschrift f. Geburtsh. u. Gynäkol. Bả. 66. S. 143.

20. Sigwart, Untersuchungen über die Hämolyse der Streptokoklen in der Schwangerschaft and im Wochenbett. Dieses Archiv. 1909. Bd. 87.

21. Heynemann, Die Bedeutung der hämolytischen Streptokokken für die puerperale Infection. Dieses Archiv. 1908. Bd. 86.

Weitere Literaturangaben finden sich in den Arbeiten der beiden letzten Autoren. 